OPEN ACCESS

Edited by:

Madalena Santos Alves,

University of Minho, Portugal

Reviewed by:

Andreas Vilcinskas,

University of Giessen, Germany

Xiaobo Liu,

Guangdong Technion-Israel Institute

of Technology, China

*Correspondence:

Jingjing Zhao

jingjing6180@126.com

Zhanyong Wang

wangzy125@gmail.com

Specialty section:

This article was submitted to Bioprocess Engineering,

a section of the journal

Frontiers in Bioengineering and

Biotechnology

Received: 04 July 2021 Accepted: 09 August 2021 Published: 18 August 2021

Citation:

Jiang S, Su T, Zhao J and Wang Z (2021) Isolation, Identification, and

Characterization of Polystyrene-

Degrading Bacteria From the Gut of

Galleria Mellonella (Lepidoptera:

Pyralidae) Larvae.

Front. Bioeng. Biotechnol. 9:736062.

doi: 10.3389/fbioe.2021.736062

\section{Isolation, Identification, and Characterization of Polystyrene-Degrading Bacteria From the Gut of Galleria Mellonella (Lepidoptera: Pyralidae) Larvae}

\author{
Shan Jiang ${ }^{1}$, Tingting Su ${ }^{1}$, Jingjing Zhao ${ }^{1 *}$ and Zhanyong Wang ${ }^{2 *}$ \\ ${ }^{1}$ School of Petrochemical Engineering, Liaoning Petrochemical University, Fushun, China, ${ }^{2}$ Department of Biotechnology, College \\ of Bioscience and Biotechnology, Shenyang Agricultural University, Shenyang, China
}

Polystyrene (PS) is a widely used petroleum-based plastic, that pollutes the environment because it is difficult to degrade. In this study, a PS degrading bacterium identified as Massilia sp. FS1903 was successfully isolated from the gut of Galleria mellonella (Lepidoptera: Pyralidae) larvae that were fed with PS foam. Scanning electron microscopy and X-ray energy dispersive spectrometry showed that the structure and morphology of the PS film was destroyed by FS 1903, and that more oxygen appeared on the degraded PS film. A water contact angle assay verified the chemical change of the PS film from initially hydrophobic to hydrophilic after degradation. X-ray photoelectron spectroscopy further demonstrated that more oxygen-containing functional groups were generated during PS degradation. After 30 days of bacterial stain incubation with $0.15 \mathrm{~g} \mathrm{PS}, 80 \mathrm{ml} \mathrm{MSM}, 30^{\circ} \mathrm{C}$ and PS of Mn 64400 and Mw $144400 \mathrm{Da}$, the weight of the PS film significantly decreased, with $12.97 \pm 1.05 \%$ weight loss. This amount of degradation exceeds or is comparable to that previously reported for other species of bacteria reported to degrade PS. These results show that Massilia sp. FS1903 can potentially be used to degrade PS waste.

Keywords: galleria mellonella, gut microbiome, polystyrene, biodegradation, massilia sp.

\section{INTRODUCTION}

Petroleum-based plastics are artificial organic polymers, obtained from natural gas or oil and used for a variety of civil and industrial applications (Suman et al., 2020). According to new data, the global output of petroleum-based plastic materials has increased to approximately 359 million tons per year, the demand for them account for approximately $80 \%$ of the total plastics used (PlasticsEurope, 2019). Polyethylene, polypropylene, polyvinyl chloride, polystyrene (PS), polyurethane and polyethylene terephthalate plastics are the main types of petroleum-based plastics (Wei and Zimmermann, 2017). Because of their profound stability and the lack of suitable degradation methods, these plastics gradually accumulate in the environment, leading to a sharp increase in environmental contamination and substantial waste (Barnes et al., 2009). Researchers have even detected plastic fragments in deep sea sediments at a depth of 5,000 m. These forms of plastic are absorbed by marine organisms, which cause serious health problems for these organisms and may also affect human health (Rochman et al., 2015; Zhang et al., 2020). 
PS, as the third most important petroleum-based plastic, is used for packaging containers, disposable cups and insulating materials, and comprises about $7 \%$ of the total amount of plastics produced (Ho et al., 2018; Urbanek et al., 2020). Because of its long persistence in the environment, PS wastes biodegradation efficiency is extremely low in natural ecosystems, and cased serious environmental pollution (Wilkes and Aristilde, 2017). Therefore, researchers around the world are exploring various PS high-efficiency degradation pathways without secondary pollution. Recently, many researchers began to investigate the biodegradation potential of intestinal microorganisms, especially in insect larvae with chewing mouthparts. Yang et al. (2015) isolated a PS-degrading bacterial strain Exiguobacterium sp. YT2 from the gut of Tenebrio molitor. This research showed that over a 30-days incubation period, YT2 formed a biofilm covering PS, resulting in obvious pits and cavities on the PS film surfaces. Over a 60-days incubation period, a suspension culture of strain YT2 degraded $7.4 \pm 0.4 \%$ of the PS pieces. Another group of researchers subsequently showed that a previously untested strain of T. molitor larvae could degrade PS (Yang et al., 2017). Moreover, Peng et al. (2019) compared the PS degradation capability of T. molitor and Tenebrio obscurus. The results demonstrated that the ability of the T. obscurus gut to degrade PS is higher than that of T. molitor and that the PS consumption rate is also greater. After 31 days of feeding, the molecular weight of residual PS in the frass of T. obscurus decreased by $26.03 \%$. Other insect species also have the same abilities as mealworms to degrade PS. Yang et al. (2020) showed that Zophobas atratus can use PS foam as their only food source, and various techniques have been used to demonstrate that the depolymerization of long-chain PS molecules and the subsequent formation of low-molecular-weight products occur in the larval gut. On the basis of this research, Pseudomonas sp., which can degrade PS, was isolated from the gut of $Z$. atratus larvae by Kim et al. (2020). Likewise, Wang et al. (2020) observed that Tribolium castaneum larvae can chew and eat PS foam, and finally successfully isolated a bacterial strain, identified as Acinetobacter sp. AnTc-1, from the gut of these larvae. Woo et al. (2020) reported PS biodegradation by the larvae of Plesiophthalmus davidis and isolated a bacterial strain Serratia sp. WSW (KCTC 82146) from their gut flora.

Galleria mellonella (Lepidoptera: Pyralidae) is a common agricultural pest that destroys the structure of honeycombs and are generally considered to be of no benefit to human beings; however, it has been discovered that G. mellonella larvae can eat and biodegrade polyethylene and PS (Lou et al., 2020). According to previous reports, this phenomenon is likely related to the gut microorganisms of these insects (Yang et al., 2015; Peng et al., 2019; Yang et al., 2020). In addition, some microbes from the gut of $G$. mellonella larvae related to the degradation of $\mathrm{PE}$ has been confirmed, and a PE degrading strain Enterobacter sp. D1 has been successfully screened (Bombelli et al., 2017; Ren et al., 2019; Cassone et al., 2020). However, there are few studies on PS degrading bacteria from the gut of G. mellonella larvae. In this study, a PS degrading bacterium was successfully isolated from the larval gut and identified by phylogenetic analysis combined with physiological and biochemical indicators. To determine the extent of PS degradation by the bacterium, the physicochemical properties of the degraded PS film were studied by a scanning electron microscope (SEM), X-ray energy dispersive spectrometer (EDS), water contact angle (WCA), and X-ray photoelectron spectroscopy (XPS) analyses.

\section{MATERIALS AND METHODS}

\section{Experimental Materials}

G. mellonella larvae were purchased from Huiyude Co. (Tianjin, China). PS foam board was obtained from Nannan Building Materials Co. (Zhejiang, China) and contained polystyrene purity over $98 \%$. The number-average molecular weight (Mn) and weightaverage molecular weight $(\mathrm{Mw})$ of the PS were 64400 and $144,400 \mathrm{Da}$, respectively, as measured by gel permeation chromatography. The PS film for microbial degradation was prepared using the previous method (Yang et al., 2015) and finetuned for this study: the foam PS was dissolved in dichloromethane $(0.03 \mathrm{~g} / \mathrm{ml})$ and then the solution was spread on a glass plate, after $5 \mathrm{~h}$, the resulting film was removed from the glass plate and immobilized in a fume hood for 3 days at room temperature. The film was then rinsed with de-ionized water and dried before use. The thickness of the prepared film was approximately $0.02 \mathrm{~mm}$.

The composition of the mineral salts medium (MSM, $\mathrm{pH}=$ 7.01) was as follows: $4.54 \mathrm{~g} / \mathrm{L} \quad \mathrm{KH}_{2} \quad \mathrm{PO}_{4}, \quad 11.94 \mathrm{~g} / \mathrm{L}$ $\mathrm{Na}_{2} \mathrm{HPO}_{4} \cdot 12 \mathrm{H}_{2} \mathrm{O}, \quad 1.0 \mathrm{~g} / \mathrm{L} \quad \mathrm{NH}_{4} \mathrm{Cl}, 0.5 \mathrm{~g} / \mathrm{L} \quad \mathrm{MgSO}_{4}, 0.005 \mathrm{~g} / \mathrm{L}$ $\mathrm{CaCl}_{2}, \quad 0.002 \mathrm{~g} / \mathrm{L} \quad \mathrm{FeSO}_{4}, \quad 0.001 \mathrm{~g} / \mathrm{L} \quad \mathrm{MnSO}_{4}$ and $0.002 \mathrm{~g} / \mathrm{L}$ $\mathrm{ZnSO}_{4}$. The Luria-Bertaini (LB) medium was prepared by dissolving $10 \mathrm{~g} \mathrm{NaCl}, 10 \mathrm{~g}$ tryptone, and $5 \mathrm{~g}$ yeast extract in $1 \mathrm{~L}$ deionized water. $15 \mathrm{~g}$ agar was added to prepare the $\mathrm{LB}$ agar medium. $0.9 \mathrm{~g} \mathrm{NaCl}$ was dissolved in $100 \mathrm{ml}$ deionized water to prepare saline. All media buffers and solutions were subjected to high-pressure steam sterilization $\left(121^{\circ} \mathrm{C}, 103.4 \mathrm{kPa}\right.$, $20 \mathrm{~min}$ ). All other chemical reagents used in this study were analytical reagent grade and obtained from commercial sources.

\section{Screening of Strains for PS Degradation Ability}

Larvae $(n=200)$ were fed with PS foam for 21 days, and then 10 larvae were collected. After sterilization, the larvae were dissected and then the intestinal tissue was placed into a $1.5 \mathrm{ml}$ centrifuge tube containing $1 \mathrm{ml}$ of normal saline, and then shaken on a vortex mixer for $5 \mathrm{~min}$. A pure intestinal cell suspension was obtained and used as a bacterial inoculum to enrich PS-degrading bacteria. A $1 \mathrm{ml}$ aliquot of the suspension was transferred into a 250-ml Erlenmeyer flask with $80 \mathrm{ml}$ MSM and $0.15 \mathrm{~g}$ PS film $(1 \times$ $3 \mathrm{~mm}$ ), which was shaken on a rotary shaker $(120 \mathrm{rpm})$ at room temperature. After 60 days, the remaining PS film was removed, and the enrichment culture was dispersed on LB agar plates. After culturing for $24 \mathrm{~h}$ at room temperature, colonies were picked and then spread on fresh LB agar plates until a pure colony was finally obtained according to the standard methodology of bacterial isolation (Yang et al., 2015). After the pure bacterial isolates were grown in liquid LB medium for $12 \mathrm{~h}$, the cells were collected by centrifugation $(10000 \mathrm{rpm})$ and then washed with sterile water 


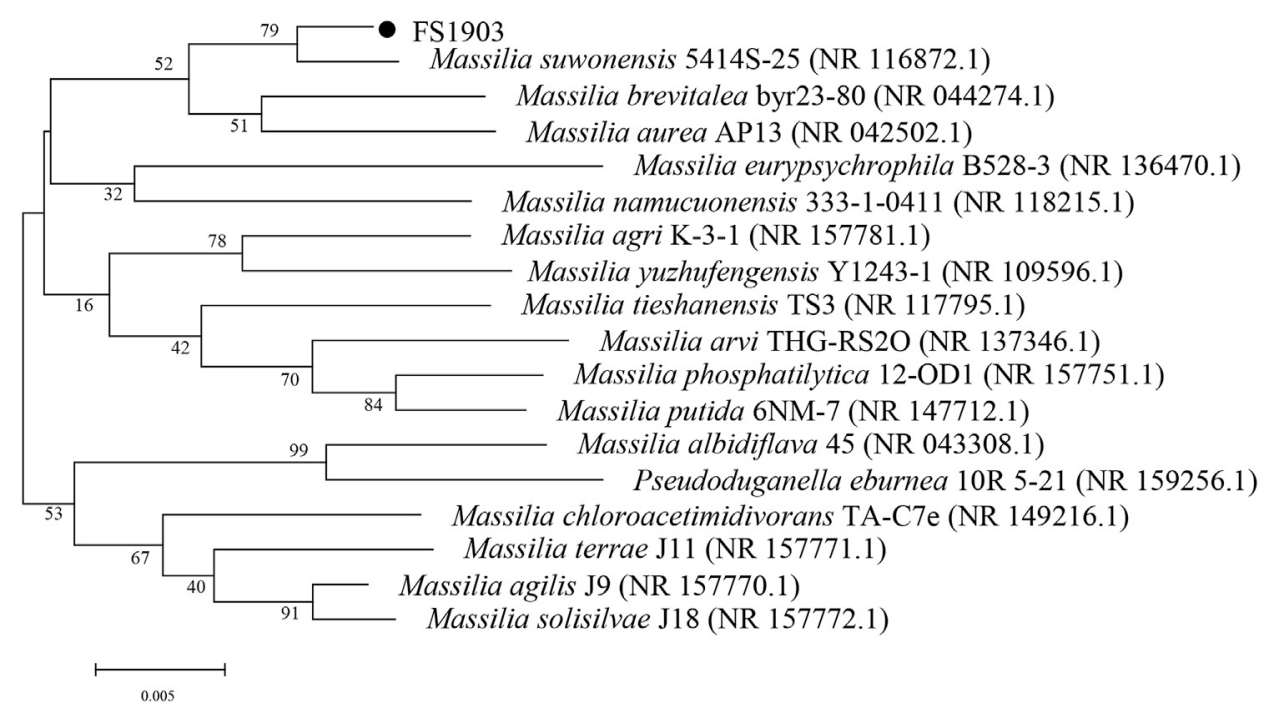

FIGURE 1 | Neighbor-joining phylogenetic tree of strain FS1903 based on the 16S rRNA gene.

to remove residual medium. This step was repeated until there were no remaining nutrients. Next, the collected cells were resuspended in sterile water and then diluted 100 times to obtain a cell suspension (Yang et al., 2015). The cell suspension $(0.4 \mathrm{ml})$ was distributed evenly on the surface of a MSM plate, which was then covered with PS film. Two control groups were set up. One was only inoculated with bacterial culture, while the other was only covered with PS film. All plates were cultured in triplicate at $30^{\circ} \mathrm{C}$ for 30 days. Place an open Petri dish with distilled water in the incubator to prevent the plates from drying. Changes in the film and bacterial growth were regularly observed. The remaining larvae were refrigerated at $4^{\circ} \mathrm{C}$ after pupation for subsequent use.

\section{Sequencing and Phylogenetic Analysis}

Genomic DNA used for 16S rDNA amplification was extracted from the cells during the logarithmic growth stage using the TIANamp Bacteria DNA Kit (Tiangen Biotech Co., Ltd., Beijing, China). Next, PCR amplification and agarose gel electrophoresis were performed to verify successful extraction. The gene was amplified using the universal primers 27-F ( $5^{\prime}$-AGAGTTTGA TCCTGGCTCAG- $\left.3^{\prime}\right)$ and 1492-R (5'-GGTTACCTTGTTACG ACTT- $\left.3^{\prime}\right)$. Amplicons in the gel were recovered using TIANquick Midi Purification Kit (Tiangen Biotech Co., Ltd., Beijing, China), and then sequenced using a kit from Sangon Biotech Co., Ltd. (Shanghai, China). The obtained sequences were aligned with known organisms in the GenBank database using the Basic Local Alignment Search Tool (BLAST) created by the National Center for Biotechnology Information (NCBI, Bethesda, MD, United States). A phylogenetic tree was constructed with MEGA5.0 software using the neighborjoining method. Bootstraps of the supporting tree branches were constructed with 1,000 replications and the default settings. A total of $1716 \mathrm{~S}$ rRNA sequences were used for the phylogenetic analyses, and the accession numbers of these sequences are shown in Figure 1.

\section{Determination of Microbial Physiological and Biochemical Indicators}

The determination of microbial physiological and biochemical indicators was carried out using the method of bacterial identification in the "Berger's Bacterial Identification Manual" (eighth edition) and Identification Manual of Common Bacteria System (Buchanna \& Gibbons 1984; Dong \& Cai 2001).

\section{Performance Analysis of the Surface of the PS Film}

An SEM (SU8010, Hitachi, Tokyo, Japan) was used to inspect the biofilm that formed on the PS film and the holes in the PS film after degradation. To observe the presence and growth of microorganisms, the PS films that covered the agar plates were removed after 30 days, fixed with $2.5 \%$ glutaraldehyde for $3 \mathrm{~h}$ and dehydrated with an ethanol gradient (30, 50, 70, 90 and 100\%) for $15 \mathrm{~min}$ (Wang et al., 2020). To detect changes in the physical and chemical properties of the PS film, the films were immersed in a $2 \%(\mathrm{w} / \mathrm{v})$ SDS aqueous solution for $4 \mathrm{~h}$, and then washed with de-ionized water to completely remove the biofilm from the surface. The PS film from the uninoculated control was also treated in the same way (Sivan et al., 2006). To verify changes in the elemental composition of the PS film surface during the degradation process, a module connected to the SEM (EDS (SU8010, Hitachi, Tokyo, Japan) was used to evaluate the differences in the composition of the carbon and oxygen elements before and after degradation.

The WCA (DSA100, KRUSS, Hamburg, Germany) was used to analyze changes in the hydrophilicity of the PS film surface. The contact angle was measured under static conditions at room temperature. The PS film was washed three times with SDS solution and then de-ionized water, and the contact angle measurement was estimated five times.

To investigate degradation and determine the variations of functional groups on the surface of the PS film, XPS (ESCALAB 


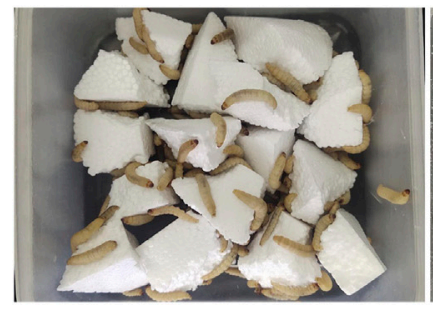

$1 d$

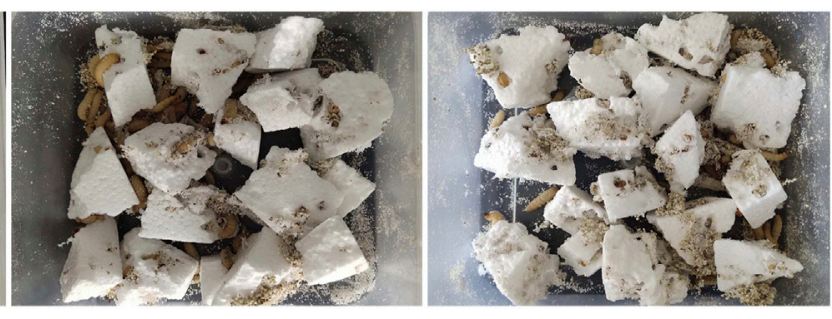

$5 \mathrm{~d}$

FIGURE 2 | Holes formed by G. mellonella larvae after feeding on PS foam for 1,3 and 5 days.

$\mathrm{Xi}+$, Thermo Fisher, Massachusetts, United States) was used to measure the binding energy. The PS film $(1 \mathrm{~cm} \times 1 \mathrm{~cm})$ was fixed on a carbon ribbon and measured within the energy range of $2+\mathrm{P}-300 \mathrm{eV}, \mathrm{C} 1 \mathrm{~s}$.

\section{Biodegradation Assay}

After overnight incubation in $4 \mathrm{ml}$ liquid LB medium, bacteria were collected by centrifugation for $10 \mathrm{~min}$ at $10,000 \mathrm{rpm}$, and then washed three times with $4 \mathrm{ml}$ of saline. PS film $(1 \mathrm{~cm} \times 3 \mathrm{~cm}, 0.15 \mathrm{~g})$ was added into a 250-ml Erlenmeyer flask containing $80 \mathrm{ml} \mathrm{MSM}$. A $1 \mathrm{ml}$ suspension of the bacterial culture was added into the flask, which was then incubated on an orbital shaker $(150 \mathrm{rpm})$ at $30^{\circ} \mathrm{C}$. This method was a slight modification of the following reported scheme (Wang et al., 2020) because the PS powder was replaced with a film; the reaction system volume was doubled; the temperature and speed were fine-tuned higher; and the reaction time was 30 days. The PS film incubated in MSM without bacteria served as a control. The weight loss assay was replicated three times. After a 30-days incubation, the PS films were harvested (Mor and Sivan, 2008).

The weight loss of the PS film was calculated using the following formula:

Percentage of weight loss $=100 \% \times$

(Initial PS weight - Final PS weight)/Initial PS weight

\section{Statistical Analysis}

Statistical ANOVAs were performed using SPSS 20.0 (SPSS Inc., Chicago, United States) to evaluate the differences in contact angle changes and weight loss produced by bacteria. Pairwise comparisons were analyzed with the student's t-test, as all date were normally distributed. All error values are reported as the mean value \pm standard deviation.

\section{RESULTS}

\section{Feeding Behavior of G. mellonella Larvae on PS Foam}

G. mellonella larvae were fed with PS for 21 days. By the third day of feeding, the PS foam block was already full of holes and a small amount of block was gnawed and attached to the culture container. By the fifth day, the whole culture container was covered with PS debris (Figure 2). After consuming the PS foam diet for 21 days, the larva appeared to be normal and they produced a normal amount of silk. These results suggested that the PS diet did not harm the larvae over a short-term feeding period.

\section{Preliminary Screening of Strains With PS Degradation Ability}

After 2 months enrichment culture, when compared with the control group, a turbid liquid was observed in the experimental group, indicating that some intestinal bacteria with the ability to degrade and use PS as a nutrition source may have been enriched. After cultivation and purification, a pure colony named as FS1903was finally obtained. When cultivated on solid LB medium, FS1903 were round with clear edges and a slightly yellowish color (Figure 3A). The shape of the bacterial cells examined with an SEM. The results showed that FS1903 cells are an elliptical rod shape approximately $1.5 \mu \mathrm{m}$ long and $0.5 \mu \mathrm{m}$ in diameter (Figure 3B). To verify the PS degrading ability of FS 1903 , a PS film $(50 \mathrm{~mm} \times 50 \mathrm{~mm})$ was supplied as the sole carbon source on top of an MSM plate that was spread with the cell suspension. After 30 days cultivation, when compared with control group (Figure 3C), some small colonies were observed under the PS film and a linear bacterial lawn appeared at the edge of the film in the experimental group (Figure 3D). This provides evidence that FS1903 isolated from the gut of G. mellonella larvae can degrade PS.

\section{Physiological and Biochemical Test of the Tested Strain}

The result of strain culturing showed that the tested strain is short rod-shaped bacterium, is motile by a terminal flagellum; forms smooth, round yellow-white colonies on LB medium; and optimum growth at $30^{\circ} \mathrm{C}$ and $\mathrm{pH}$ 7.0. Physiological and biochemical characteristics of the strain were list at Table 1. According to the physiological and biochemical characteristics results, the strain is similar to the microorganisms of the genus Massilia (Shen et al., 2013; Orthova et al., 2015). In addition, bacteria of this family are often isolated from the rumen or the large intestine of humans and animals (Kämpfer et al., 2012), this is also in line with our research. 

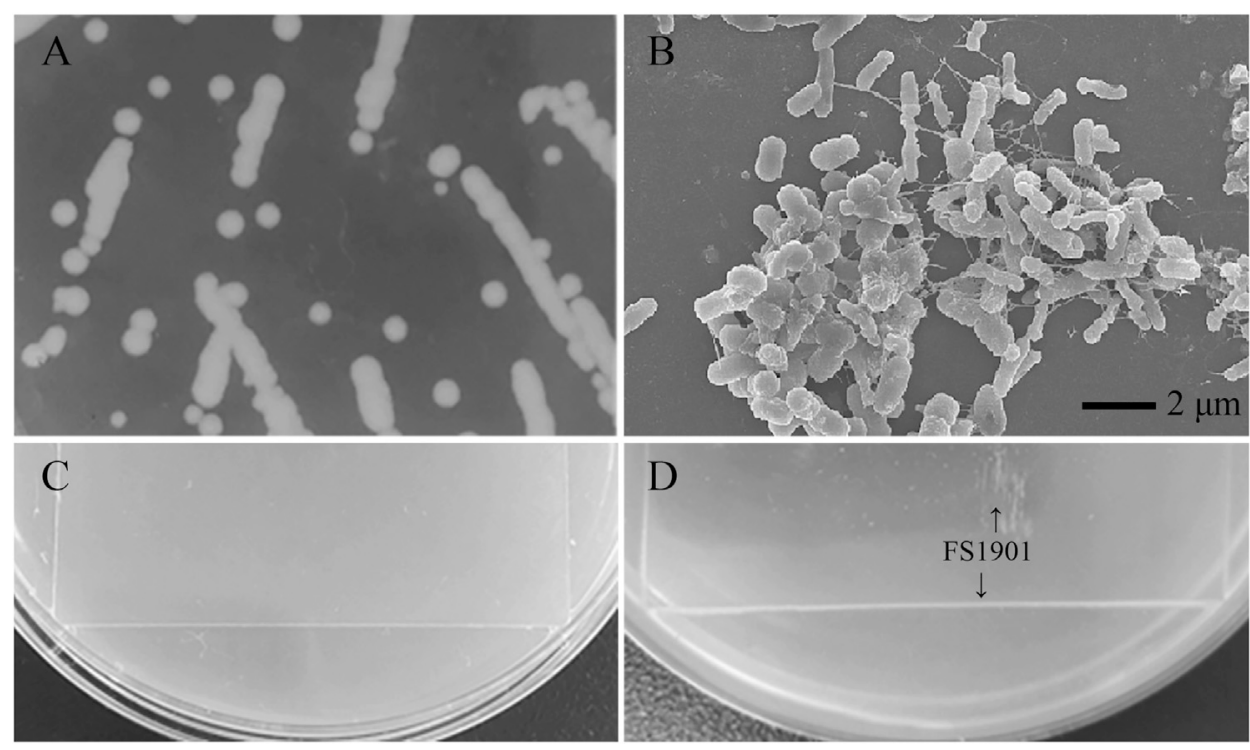

FIGURE 3 | Colonies of FS1903 on LB agar plates (A); SEM of FS 1903 (B); the control (C); and the colonies and lawn of FS1903 on the PS film on the MSM plate (D)

\section{Sequencing and Phylogenetic Tree Construction of FS1903}

A phylogenetic tree of FS1903 and other Massilia bacteria was constructed based on the 16S rRNA gene (Figure 1). According to the phylogenetic tree, FS1903 and Massilia suwonensis 5414s25 were on the same branch and closely related with high sequence similarity of $79 \%$. Combined with the physiological and biochemical test, tested strain can be identified as the genus Massilia, and then the strain was named Massilia sp. FS 1903. The $16 \mathrm{~S}$ rRNA gene sequence of this bacterium was deposited in the GenBank database under accession number MW138062.

\section{Observation of the Biofilm Formed on the PS Film Surface}

The biofilm was scanned with an SEM to more closely examine the effects of the colonization of the PS film surface by FS 1903. After 30 days of cultivation, the PS film surface was examined before and after the microorganisms were completely washed off, and the results showed that the PS film was damaged by the bacteria (Figure 4A,B). In contrast, the surface of the uncultured control was smooth (Figure 4C) without any defects. These observations revealed that the bacteria caused pits and cavities on the film surface. The maximum width of a typical cavity was approximately $4 \mu \mathrm{m}$ (Figure 4D).

\section{Analysis of the Composition of the PS Film Surface}

The carbon and oxygen composition on the PS surface where the bacteria grew was analyzed by EDS to determine the effects of the bacteria on the composition of the film. There were no obvious
TABLE 1 | Physiological and biochemical characteristics of strain FS1903.

Characteristic

FS1903

Maltose

Glucose

Lactose

Rhamnose

Sucrose

Arabinose

Starch

Esculin

Sorbitol

Inositol

L-tyrosine hydrolysis

$\mathrm{V}-\mathrm{P}(2 \mathrm{~d})$

$\mathrm{V}-\mathrm{P}$ (6d)

Contact enzyme

Propionate

gelatin liquefaction

differences in the number of carbon atoms between the PS in the control group and the experimental group (Figure 5). However, more oxygen atoms were detected in the experimental group, which shows that oxidization occurred.

Oxidation was confirmed by measuring changes in the contact angle of water droplets on the PS surface, which is indicative of the surface hydrophobicity of the PS film. The results show that the contact angle of the experimental group was $66.1 \pm 5.1^{\circ}$, while that of the control group was $96.0 \pm 3.8^{\circ}$ (Figure 6). Compare to the control, the contact angle of the experimental group was significantly decreased $(p<0.01)$. This decrease in the contact angle shows that the surface tension of water decreased, because of the insertion of oxygen on the surface of PS during the oxidation 

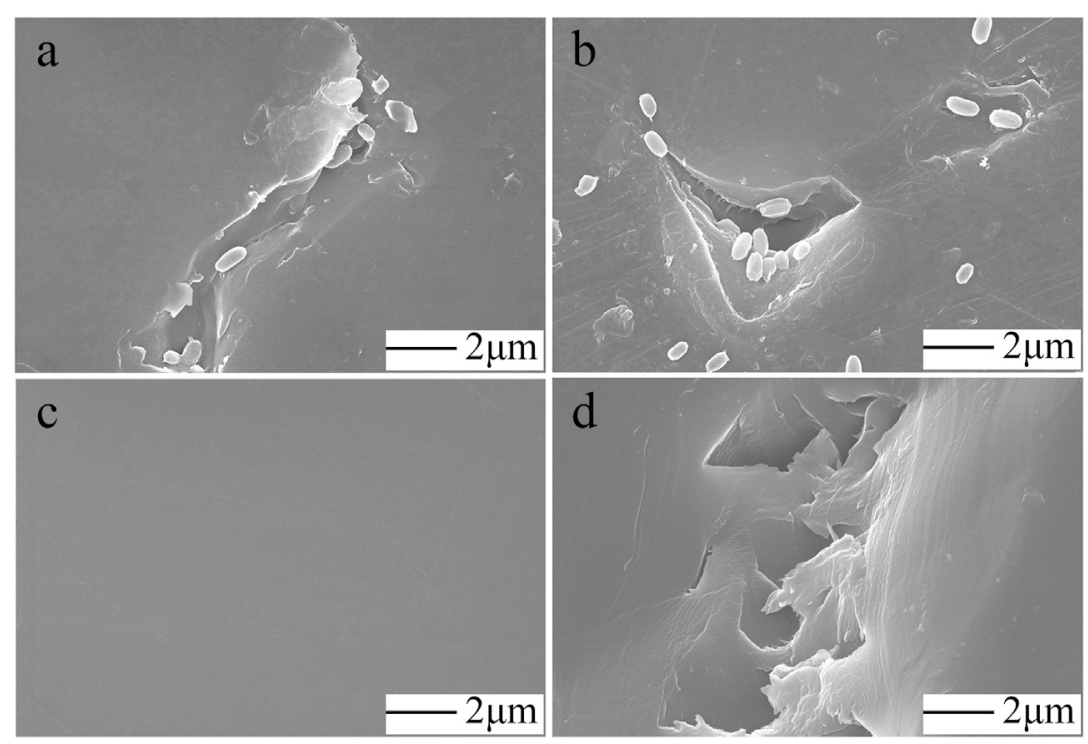

FIGURE 4 | SEM observations. FS1903 cells on the degraded PS film (A, B), control (C) and cavities and pits on the surface caused by incubation with FS 1903 (D).

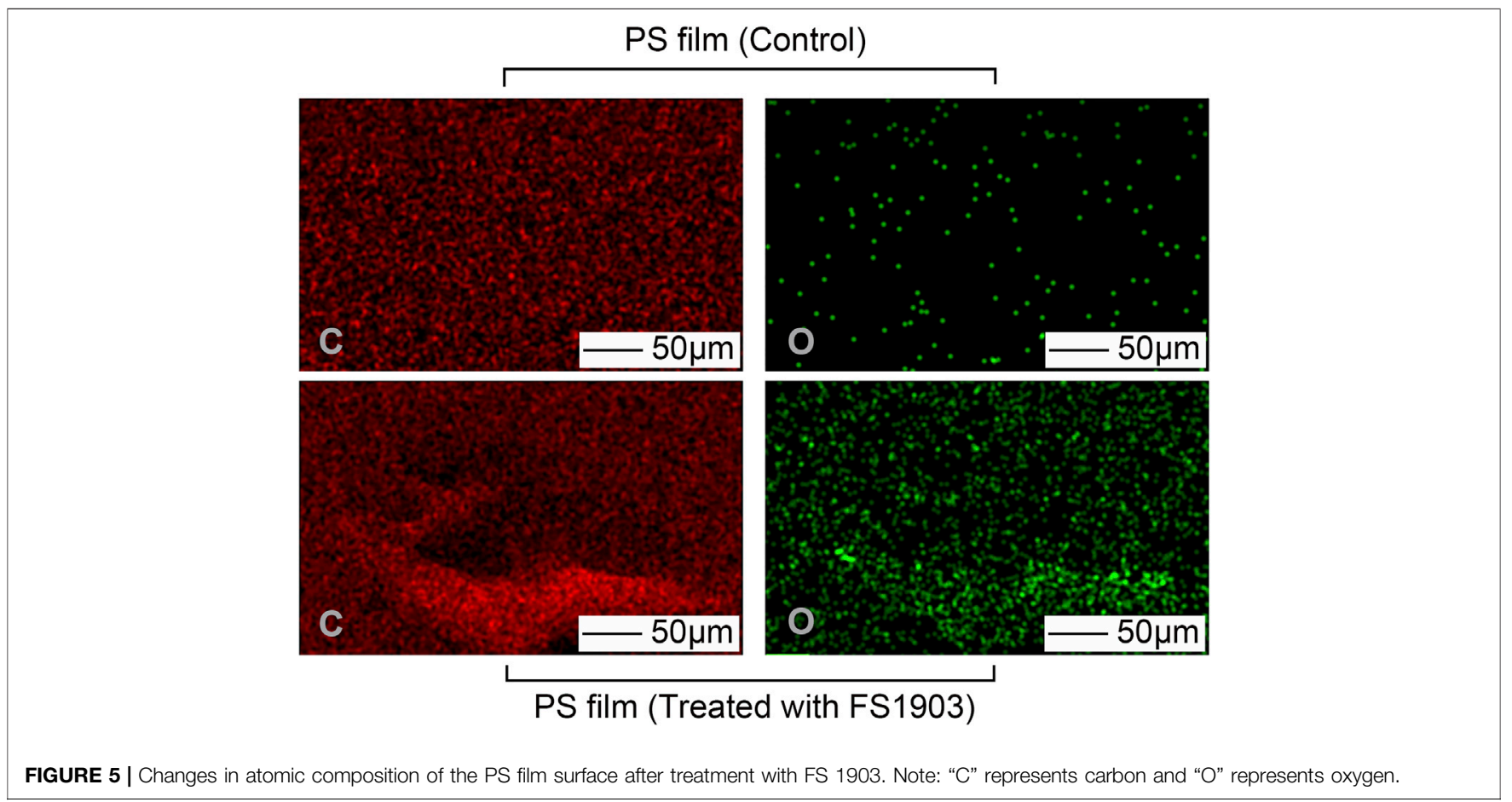

process. Oxidation during PS degradation converts hydrophobic regions to hydrophilic ones, thereby changing the chemical properties of the PS surface (Gu, 2003). FS1903 colonization reduced the hydrophobicity of the PS film and at the same time the film was damaged, the decreased hydrophobicity reduced the resistance to bacterial cells to subsequent PS degradation.

XPS was used to analyze changes in the surface chemical composition and functional groups. Figure 7 shows the comparison $(0-900 \mathrm{eV})$ of the XPS scanning spectra of the PS film for the inoculated experimental and non-inoculated control groups. The control group had only surface carbon at peak of $284.8 \mathrm{eV}$, while the PS inoculated with strain FS 1903 except $284.8 \mathrm{eV}$ had another obvious peak at $532.3 \mathrm{eV}$ that represented the amount of surface oxygen (Figure 7A). The XPS spectra of C1s on the PS film surface inoculated with strain FS1903 versus the control was compared (Figure 7B), and it showed that culturing with the FS1903 caused a notable decrease of the C-C group $(284.8 \mathrm{eV})$. Meanwhile, compare to the control 


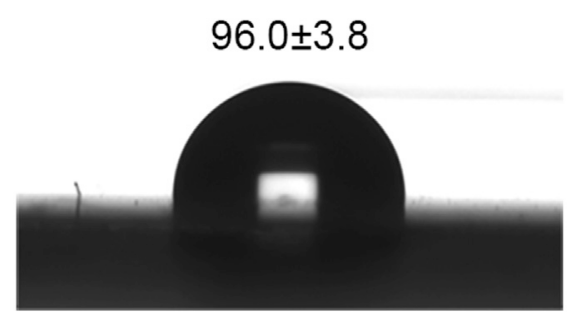

PS film

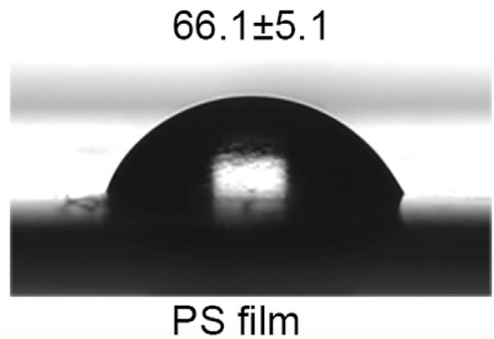

(Treated with FS1903)

FIGURE 6 | WCA of PS film without or with inoculation by strain FS1903.
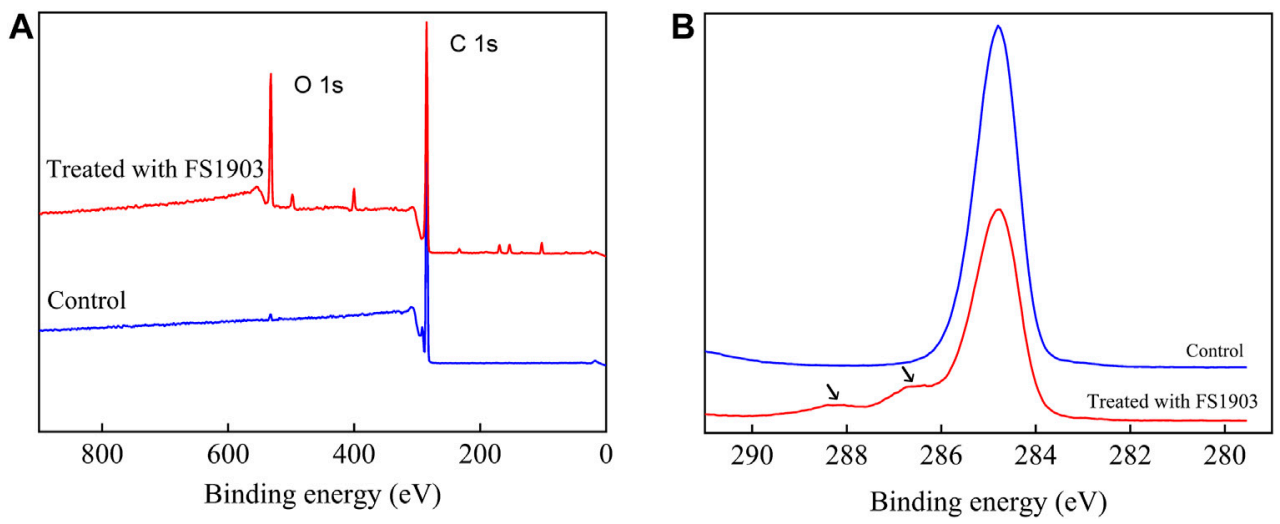

FIGURE 7 | XPS scanning (A) and C1s spectra (B) of the control and the residual PS films inoculated with FS1903.

group with one peak, the PS inoculated with strain FS 1903 had another peak at $286.5 \mathrm{eV}$, which was assigned to the $\mathrm{C}-\mathrm{O}$ group. This implies that part of the $\mathrm{C}-\mathrm{C}$ groups in the PS was oxidized to alcohol and carboxylic-acid-like compounds (Shang et al., 2003). In addition, on the PS film surface inoculated with strain FS 1903, another new peak appeared at $288 \mathrm{eV}$, which was assigned to the $-\mathrm{C}=\mathrm{O}$ group. These observations all demonstrate a transition from a $\mathrm{C}-\mathrm{C}$ bond to $-\mathrm{C}=\mathrm{O}$ and $\mathrm{C}-\mathrm{O}$ bonds during the degradation process. Furthermore, these results indicate that strain FS1903 is capable of attacking or oxidizing the PS structure to produce more polar derivatives. This change in binding energy confirms that oxidation occurs during FS1903-mediated degradation. The results are in accordance with previous work (Yang et al., 2015; Kim et al., 2020).

\section{Biodegradation Assay}

To confirm the degradation ability of strain FS 1903, we calculated the weight loss after infestation with the bacteria. After cultivation with the bacteria for 30 days, the weight loss of PS treated with strain FS1903 was $12.97 \pm 1.05 \%$, which was significantly decreased compared with the control group $(p<0.01)$. Furthermore, FS1903 degraded the PS without being supplied with nutritional supplements, such as yeast extract or gelatin.

\section{DISCUSSION}

According to previous literature (Yang et al., 2015; Yang et al., 2018; Peng et al., 2019), using antibiotics to inhibit gut microorganisms of certain insects that can feed on plastic directly leads to the loss of the insects' ability to degrade plastic. At the same time, microorganisms that can degrade plastic polyethylene or PS from insect intestinal microbiomes were isolated (Yang et al., 2015; Kim et al., 2020; Wang et al., 2020). Therefore, it is clear that plastic-degrading bacteria do exist in the gut of some insects. Since the G. mellonella larvae have the ability to eat polystyrene and still grow normally, we preliminarily speculated that there may be microorganisms that can digest PS in the gut of these larvae. Our results confirmed this hypothesis and screened a strain of PS degrading bacteria.

The most notable and fundamental characteristic of bacteriamediated plastic degradation processes is the formation of a biofilm on the plastic surface (Kim et al., 2020). Biofilms form on a substrate surface and can further penetrate into the materials to erode and deteriorate them (Chauhan et al., 2018; Liu et al., 2020). Adherence of bacterial cells to the polymer surface is the first and most basic step of subsequent biodegradation (Esmaeili et al., 2013). In this study, the bacterial strain FS 1903 can form a biofilm on PS film. After the biofilm is formed, PS is oxidized. 
Oxidation, transform the PS surface from hydrophobic to hydrophilic, is a key step in PS biodegradation (Tribedi and Sil, 2014). The oxidation in the experimental group is attributed to the functional groups (such as hydroxyl or carbonyl groups) formed via $\beta$-oxidation (Bode et al., 2000). This phenomenon caused by an enzyme or a variety of enzymes secreted by bacterial cells attaching to PS film that promotes the degradation and oxidation of PS (Mohanan et al., 2020). According to the analysis results of the composition, contact angle, and functional groups of the PS film surface, oxidation was produced in the PS films treated by FS 1903. These results confirmed that FS1903 can destroy the physical integrity of a PS film and degrade it.

Although the physics and chemistry changes in the PS inoculated with the bacteria demonstrate the degradation by FS 1903, the most direct evidence of PS biodegradation is its weight loss (Yang et al., 2015; Kim et al., 2020; Wang et al., 2020). Rhodococcus C208 and Exiguobacterium sp. YT2 have been previously shown to reduce the PS weight by $0.8 \%$ in 56 days and $7.4 \%$ in 60 days, respectively (Mor and Sivan, 2008; Yang et al., 2017). Acinetobacter sp. AnTc-1 can degrade PS powder, with a weight loss of $12.14 \pm 1.4 \%$ after 60 days of cultivation (Wang et al., 2020). Compared to these other bacterial strains, FS1903 produced a higher weight loss in less time and demonstrated a comparable potential in degrading PS. However, the weight loss is very low compared to the direct consumption of PS by insect larvae. Previous researches have reported that plastic-degrading microorganisms demonstrate expedited degradation within the insect gut environment (Przemieniecki et al., 2020; Brandon et al., 2021). This phenomenon implies that the insect host may play a role in the plastic biodegradation process. G. mellonella larvae have been shown that they retain the capacity to metabolize polyethylene when their gut microbiome activity is suppressed (Kong et al., 2019). In addition, Brandon et al. (2021) and Tsochatzis et al. (2021) provide evidence that insect larvae secrete emulsifying factors or enzymes that mediate plastic biodegradation. These studies indicate that using insect larvae to degrade plastic is a complex system in which the larvae host and its gut microbiome collaborate to work. This may be the reason for the low weight loss of PS degradation by FS1903 only. Therefore, further identification key functional enzymes from larvae host and gut microbiome related to the depolymerization and biodegradation of PS is needed.

\section{REFERENCES}

Barnes, D. K. A., Galgani, F., Thompson, R. C., and Barlaz, M. (2009). Accumulation and Fragmentation of Plastic Debris in Global Environments. Phil. Trans. R. Soc. B 364, 1985-1998. doi:10.1098/rstb.2008.0205

Bode, H. B., Zeeck, A., Plückhahn, K., and Jendrossek, D. (2000). Physiological and Chemical Investigations into Microbial Degradation of Synthetic Poly( Cis -1,4-isoprene). Appl. Environ. Microbiol. 66, 3680-3685. doi:10.1128/AEM.66.9.3680-3685.2000

Bombelli, P., Howe, C. J., and Bertocchini, F. (2017). Polyethylene Bio-Degradation by Caterpillars of the Wax Moth Galleria Mellonella. Curr. Biol. 27, R292-R293. doi:10.1016/j.cub.2017.02.060

\section{CONCLUSION}

This is the first study to identify a PS-degrading strain of bacteria isolated from the gut of G. mellonella larvae. The degradation ability of this strain FS1903 was comparable or better than any other bacterial strain previously identified. Optimization of conditions to simulate the intestinal environment and improve the degradation efficiency of these bacteria in vitro is currently being investigated in our laboratory. In addition, further work is needed to determine if the larvae have the ability to degrade other common plastics (such as polyethylene, polypropylene, polyvinyl chloride and polyethylene terephthalate), and to identify the mechanisms and pathways involved in this biodegradation.

\section{DATA AVAILABILITY STATEMENT}

The datasets presented in this study can be found in online repositories. The names of the repository/repositories and accession number(s) can be found below: https://www.ncbi. nlm.nih.gov/nuccore/MW138062.1.

\section{ETHICS STATEMENT}

The animal study was reviewed and approved by International Association of Veterinary Editors guidelines.

\section{AUTHOR CONTRIBUTIONS}

SJ, TS, JZ and ZW: conceptualization. SJ: methodology, data curation. SJ and TS: formal analysis. SJ and JZ: writing-original draft. JZ and ZW: writing-review and editing. All authors contributed to manuscript revision, read, and approved the submitted version.

\section{FUNDING}

This work was supported by the Education Department of Liaoning Province (L2019040); the Natural Science Foundation of Liaoning Province (2020-BS-229); Liaoning Revitalization Talents Program (XLYC1807034); the Talent Scientific Research Funds of Liaoning Petrochemical University (2019XJJL-012).

Brandon, A. M., Garcia, A. M., Khlystov, N. A., Wu, W.-M., and Criddle, C. S. (2021). Enhanced Bioavailability and Microbial Biodegradation of Polystyrene in an Enrichment Derived from the Gut Microbiome of Tenebrio molitor (Mealworm Larvae). Environ. Sci. Technol. 55 (3), 2027-2036. doi:10.1021/ acs.est.0c04952

Buchanna, R. E., and Gibbons, N. E. (1984). Bergey's Manual of Determinative Bacteriology. The Eighth Edition. Beijing: Science Press.

Cassone, B. J., Grove, H. C., Elebute, O., Villanueva, S. M. P., and Lemoine, C. M. R. (2020). Role of the Intestinal Microbiome in Low-Density Polyethylene Degradation by Caterpillar Larvae of the Greater Wax Moth, Galleria Mellonella. Proc. R. Soc. B. 287, 20200112. doi:10.1098/ rspb.2020.0112 
Chauhan, D., Agrawal, G., Deshmukh, S., Roy, S. S., and Priyadarshini, R. (2018). Biofilm Formation byExiguobacteriumsp. DR11 and DR14 Alter Polystyrene Surface Properties and Initiate Biodegradation. RSC Adv. 8, 37590-37599. doi:10.1039/C8RA06448B

Dong, X. Z., and Cai, M. Y. (2001). Manual for Systematic Identification of Common Bacteria. Beijing: Science Press.

Esmaeili, A., Pourbabaee, A. A., Alikhani, H. A., Shabani, F., and Esmaeili, E. (2013). Biodegradation of Low-Density Polyethylene (LDPE) by Mixed Culture of Lysinibacillus Xylanilyticus and Aspergillus niger in Soil. PLoS One 8, e71720. doi:10.1371/journal.pone.0071720

Gu, J.-D. (2003). Microbiological Deterioration and Degradation of Synthetic Polymeric Materials: Recent Research Advances. Int. Biodeterior. Biodegrad. 52, 69-91. doi:10.1016/S0964-8305(02)00177-4

Ho, B. T., Roberts, T. K., and Lucas, S. (2018). An Overview on Biodegradation of Polystyrene and Modified Polystyrene: the Microbial Approach. Crit. Rev. Biotechnol. 38, 308-320. doi:10.1080/07388551.2017.1355293

Kämpfer, P., Lodders, N., Martin, K., and Falsen, E. (2012). Massilia Oculi Sp. nov., Isolated from a Human Clinical Specimen. Int. J. Stst. Evo.l Micr. 62 (Pt 2), 364-369. doi:10.1099/ijs.0.032441-0

Kim, H. R., Lee, H. M., Yu, H. C., Jeon, E., Lee, S., Li, J., et al. (2020). Biodegradation of Polystyrene by Pseudomonas Sp. Isolated from the Gut of Superworms (Larvae of Zophobas Atratus). Environ. Sci. Technol. 54, 6987-6996. doi:10.1021/acs.est.0c01495

Kong, H. G., Kim, H. H., Chung, J.-h., Jun, J., Lee, S., Kim, H.-M., et al. (2019). The Galleria Mellonella Hologenome Supports Microbiota-independent Metabolism of Long-Chain Hydrocarbon Beeswax. Cel Rep. 26, 2451-2464. doi:10.1016/j.celrep.2019.02.018

Liu, X., Koestler, R. J., Warscheid, T., Katayama, Y., and Gu, J.-D. (2020). Microbial Deterioration and Sustainable Conservation of Stone Monuments and Buildings. Nat. Sustain. 3, 991-1004. doi:10.1038/s41893-020-00602-5

Lou, Y., Ekaterina, P., Yang, S.-S., Lu, B., Liu, B., Ren, N., et al. (2020). Biodegradation of Polyethylene and Polystyrene by Greater Wax Moth Larvae (Galleria Mellonella L.) and the Effect of Co-diet Supplementation on the Core Gut Microbiome. Environ. Sci. Technol. 54, 2821-2831. doi:10.1021/acs.est.9b07044

Mohanan, N., Montazer, Z., Sharma, P. K., and Levin, D. B. (2020). Microbial and Enzymatic Degradation of Synthetic Plastics. Front. Microbiol. 11, 580709. doi:10.3389/fmicb.2020.580709

Mor, R., and Sivan, A. (2008). Biofilm Formation and Partial Biodegradation of Polystyrene by the Actinomycete Rhodococcus Ruber. Biodegradation 19, 851-858. doi:10.1007/s10532-008-9188-0

Orthová, I., Kämpfer, P., Glaeser, S. P., Kaden, R., and Busse, H.-J. (2015). Massilia Norwichensis Sp. nov., Isolated from an Air Sample. Int. J. Syst. Evol. Micr. 65, 56-64. doi:10.1099/ijs.0.068296-0

Peng, B.-Y., Su, Y., Chen, Z., Chen, J., Zhou, X., Benbow, M. E., et al. (2019). Biodegradation of Polystyrene by Dark (Tenebrio Obscurus) and Yellow (Tenebrio Molitor) Mealworms (Coleoptera: Tenebrionidae). Environ. Sci. Technol. 53, 5256-5265. doi:10.1021/acs.est.8b06963

PlasticsEurope (2019). Plastics-the Facts 2019. An Analysis of European Plastics Production, Demand and Waste Data. Available at: https://www.plasticseurope. org/en/resources/publications/1804-plastics-facts-2019 (Accessed October 17, 2019).

Przemieniecki, S. W., Kosewska, A., Ciesielski, S., and Kosewska, O. (2020). Changes in the Gut Microbiome and Enzymatic Profile of Tenebrio Molitor Larvae Biodegrading Cellulose, Polyethylene and Polystyrene Waste. Environ. Pollut. 256, 113265. doi:10.1016/j.envpol.2019.113265

Ren, L., Men, L., Zhang, Z., Guan, F., Tian, J., Wang, B., et al. (2019). Biodegradation of Polyethylene by Enterobacter Sp. D1 from the Guts of Wax Moth Galleria Mellonella. Ijerph 16, 1941. doi:10.3390/ijerph16111941

Rochman, C. M., Tahir, A., Williams, S. L., Baxa, D. V., Lam, R., Miller, J. T., et al. (2015). Anthropogenic Debris in Seafood: Plastic Debris and Fibers from Textiles in Fish and Bivalves Sold for Human Consumption. Sci. Rep. 5, 14340. doi: $10.1038 /$ srep 14340

Shang, J., Chai, M., and Zhu, Y. (2003). Solid-phase Photocatalytic Degradation of Polystyrene Plastic with TiO2 as Photocatalyst. J. Solid State. Chem. 174, 104-110. doi:10.1016/S0022-4596(03)00183-X

Shen, L., Liu, Y., Wang, N., Yao, T., Jiao, N., Liu, H., et al. (2013). Massilia Yuzhufengensis Sp. nov., Isolated from an Ice Core. Int. J. Syst. Evol. Micr. 63, 1285-1290. doi:10.1099/ijs.0.042101-0
Sivan, A., Szanto, M., and Pavlov, V. (2006). Biofilm Development of the Polyethylene-Degrading Bacterium Rhodococcus Ruber. Appl. Microbiol. Biotechnol. 72, 346-352. doi:10.1007/s00253-005-0259-4

Suman, T. Y., Li, W.-G., Alif, S., Faris, V. R. P., Amarnath, D. J., Ma, J.-G., et al. (2020). Characterization of Petroleum-Based Plastics and Their Absorbed Trace Metals from the Sediments of the Marina Beach in Chennai, India. Environ. Sci. Eur. 32, 110. doi:10.1186/s12302-020-00388-5

Tribedi, P., and Sil, A. K. (2014). Cell Surface Hydrophobicity: A Key Component in the Degradation of Polyethylene Succinate by Pseudomonas Sp. AKS2. J. Appl. Microbiol. 116, 295-303. doi:10.1111/jam.12375

Tsochatzis, E. D., Berggreen, I. E., Nørgaard, J. V., Theodoridis, G., and Dalsgaard, T. K. (2021). Biodegradation of Expanded Polystyrene by Mealworm Larvae under Different Feeding Strategies Evaluated by Metabolic Profiling Using GC-TOFMS. Chemosphere 281, 130840. doi:10.1016/j.chemosphere.2021.130840

Urbanek, A. K., Rybak, J., Wróbel, M., Leluk, K., and Mirończuk, A. M. (2020). A Comprehensive Assessment of Microbiome Diversity in Tenebrio molitor Fed with Polystyrene Waste. Environ. Pollut. 262, 114281. doi:10.1016/j.envpol.2020.114281

Wang, Z., Xin, X., Shi, X., and Zhang, Y. (2020). A Polystyrene-Degrading Acinetobacter Bacterium Isolated from the Larvae of Tribolium castaneum. Sci. Total Environ. 726, 138564. doi:10.1016/j.scitotenv.2020.138564

Wei, R., and Zimmermann, W. (2017). Microbial Enzymes for the Recycling of Recalcitrant Petroleum-based Plastics: How Far Are We? Microb. Biotechnol. 10, 1308-1322. doi:10.1111/1751-7915.12710

Wilkes, R. A., and Aristilde, L. (2017). Degradation and Metabolism of Synthetic Plastics and Associated Products byPseudomonassp.: Capabilities and Challenges. J. Appl. Microbiol. 123, 582-593. doi:10.1111/jam.13472

Woo, S., Song, I., and Cha, H. J. (2020). Fast and Facile Biodegradation of Polystyrene by the Gut Microbial flora of Plesiophthalmus Davidis Larvae. Appl. Environ. Microbiol. 86, e01361-20. doi:10.1128/AEM.01361-20

Yang, Y., Yang, J., Wu, W.-M., Zhao, J., Song, Y., Gao, L., et al. (2015). Biodegradation and Mineralization of Polystyrene by Plastic-Eating Mealworms: Part 2. Role of Gut Microorganisms. Environ. Sci. Technol. 49, 12087-12093. doi:10.1021/acs.est.5b02663

Yang, S.-S., Brandon, A. M., Andrew Flanagan, J. C., Yang, J., Ning, D., Cai, S.-Y., et al. (2018). Biodegradation of Polystyrene Wastes in Yellow Mealworms (Larvae of Tenebrio molitor Linnaeus): Factors Affecting Biodegradation Rates and the Ability of Polystyrene-Fed Larvae to Complete Their Life Cycle. Chemosphere 191, 979-989. doi:10.1016/j.chemosphere.2017.10.117

Yang, S.-S., Wu, W.-M., Brandon, A. M., Fan, H.-Q., Receveur, J. P., Li, Y., et al. (2018). Ubiquity of Polystyrene Digestion and Biodegradation within Yellow Mealworms, Larvae of Tenebrio molitor Linnaeus (Coleoptera: Tenebrionidae). Chemosphere 212, 262-271. doi:10.1016/j.chemosphere.2018.08.078

Yang, Y., Wang, J., and Xia, M. (2020). Biodegradation and Mineralization of Polystyrene by Plastic-Eating Superworms Zophobas Atratus. Sci. Total Environ. 708, 135233. doi:10.1016/j.scitotenv.2019.135233

Zhang, D., Liu, X., Huang, W., Li, J., Wang, C., Zhang, D., et al. (2020). Microplastic Pollution in Deep-Sea Sediments and Organisms of the Western Pacific Ocean. Environ. Pollut. 259, 113948. doi:10.1016/j.envpol.2020.113948

Conflict of Interest: The authors declare that the research was conducted in the absence of any commercial or financial relationships that could be construed as a potential conflict of interest.

Publisher's Note: All claims expressed in this article are solely those of the authors and do not necessarily represent those of their affiliated organizations, or those of the publisher, the editors and the reviewers. Any product that may be evaluated in this article, or claim that may be made by its manufacturer, is not guaranteed or endorsed by the publisher.

Copyright (c) 2021 Jiang, Su, Zhao and Wang. This is an open-access article distributed under the terms of the Creative Commons Attribution License (CC $B Y$ ). The use, distribution or reproduction in other forums is permitted, provided the original author(s) and the copyright owner(s) are credited and that the original publication in this journal is cited, in accordance with accepted academic practice. No use, distribution or reproduction is permitted which does not comply with these terms. 